\title{
Extracting Linear Features from SAR Images Using CGVF Snake Model and Beamlet Transform
}

\author{
V. Ramachandran and K. Vani \\ Department of Information Science and Technology \\ CEG, Anna University, Chennai, India \\ ramendrn@gmail.com, \\ vani@annauniv.edu
}

\begin{abstract}
Extracting Linear Features from Microwave Images (SAR) using CGVF Snake Model and Beamlet Transform is proposed in this paper. Microwave images are independent of climate. In certain durations these images are affected by noise. So noise is removed in this work. For CGVF Snake model, edges are detected and GVF field is produced followed by Snake is initialized and then two external constraint forces are developed. The first one will points on the snake and determine the basic shape of a snake. The second one generating the curves is smooth and grows in a correct direction. For each iteration, the snake is deformed in edges and removes the discontinuities for extracting the linear features. For beamlet Transform, initially the SAR image is enhanced by applying the Non-uniform Background elimination method followed by Beamlet Transform based algorithm was applied. This algorithm recursively partitions the image into sub-squares to build a beamlet dictionary to perform the transform followed by discontinuities are linked to extract the linear features. The results of feature extraction from microwave images using CGVF Snake model and Beamlet Transform to increase the correctness and quality of satellite Mapping.
\end{abstract}

Keywords: gradient vector flow (GVF), synthetic aperture radar (SAR), controllable gradient vector flow (CGVF), Beamlet Transform, linear feature extraction.

\section{Introduction}

Microwave images(SAR) are capable of operating under weather conditions and have more noise(i.e. Salt and pepper noise and also speckle noise) .These images are suitable options for most of the real time application such as road extraction, ridge extraction etc. Due to noisy nature, SAR image is not a suitable option for real time application, so proper pre-processing is mandatory option for SAR image.

Removing noise in the image needs the filter technique such as Median filter [7]. Median filter helps to reduce the conflict occurs due to presence of salt and pepper noise and the speckle noise is to be reduced by thresholding technique. Because of 
curve Nature, Most of the edge detection technique will not suitable for extracting the edges of the road.

After removing the noise, edges are detected. Normally the Edge in an image may be defined as a rapid photometric change in the registered intensity values. From a pixel level perspective, edge can be viewed as the regions of an image where the image values undergo a sharp variation. In discrete 2-D image edge is detected as linear combination of the point's singularities between pixels. The edge detection is discussed in [3].In proposed work canny edge detection is applied to detect the edges followed by feature is extracted.

Microwave images contain many linear features (i.e. road and ridge networks). Extracting those linear features is a fundamental task in many image processing and computer vision applications. Traditionally the snakes are developed to detect features. In [1], M. Kass and A. Witkin presents Snake is a curve defined within an image domain that can move under the influence of internal forces coming from the curves themselves and external forces computed from the image data. In [2], Xu et al. present a class of external force named gradient vector flow (GVF) that largely solves the problems such as initialization and poor convergence to boundary concavities of traditional snakes. In particular, they may be not pulled to the target feature correctly.

Aiming at improving the performance of traditional GVF snakes, the improved CGVF snakes are employed in this work to close gaps in linear feature extraction which is important step involved. Further improve the feature extraction process beamlet transform is proposed in this paper. In [11], XiaoYu DOU HongXun SONG presents the beamlet Transform based algorithm to detect the cracks in image. also E.Salari and Y.Zhu [7] presents the road extraction using the Beamlet transform. But they also contain some discontinuities. But they also contain some discontinuities.

In proposed Beamlet Transform process, the image is be enhanced [6] followed by recursively partitioned into sub-squares to build the beamlet dictionary and find the beamlet Transform co-efficient to construct the beamlet image. The discontinuities present in the beamlet image are linked efficiently by using Thinning method. Finally compare the efficiency of CGVF Snake Model and Beamlet Transform method to find the best approach for extracting the linear feature in efficient manner.

The rest of the paper is organized as follows Section 2 contains the system description. It describes the overall system contains noise removal, edge detection and linear Feature Extraction using CGVF Snake Model, Image Enhancement, Linear Feature Extraction Using Beamlet Transform and Performance evaluation. Section 3 discusses experimental results and finally Conclusion is given in Section 6.

\section{System Description}

The System Architecture for linear feature extraction from microwave images using CGVF Snake Model and Beamlet Transform is illustrated in figure1. 


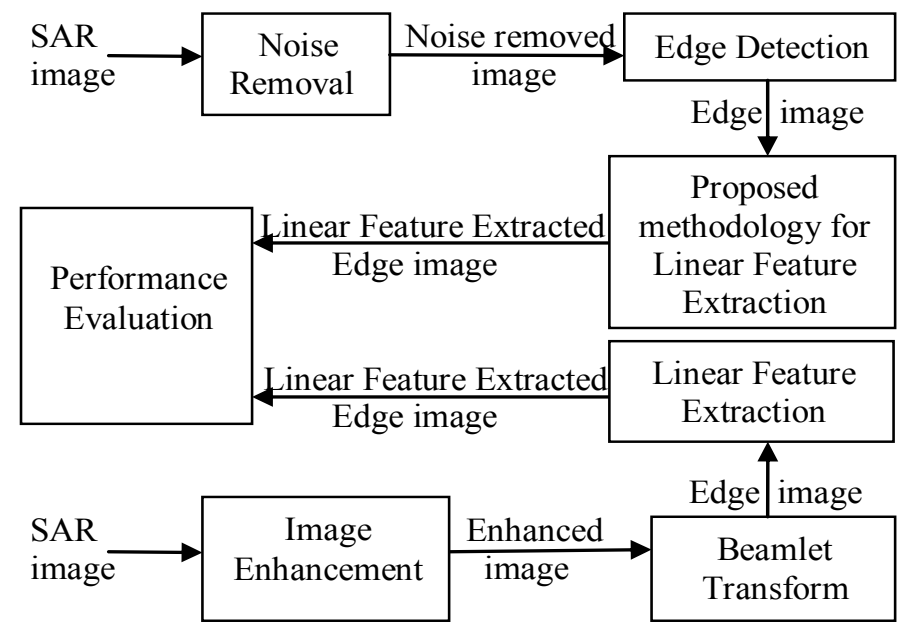

Fig. 1. System Architecture

Each process of the system is to be constructed and described below.

\section{A. Noise removal}

Presence of noise in an image is the major drawback for SAR image. It shows false edges in the Edge mapped image. So keeping Noise Removal process prior to all is an effective idea is illustrated in figure 2.

Initially Noisy (SAR) image is converted to gray scale image is followed by Histogram equalization to enhance the contrast and median filter helps to remove the salt and pepper noise and finally produces the noise removed image.

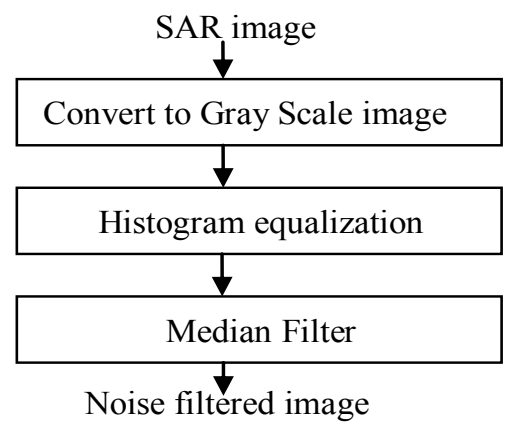

Fig. 2. Noise Removal

1) Convert to Gray Scale image: It converts RGB to Gray scale by replacing the RGB values.

$$
Y=w R \boldsymbol{R}+w G \boldsymbol{G}+w B \boldsymbol{B}
$$

Where R, G, B and Y are Red, Green, Blue and Gray pixel . wR, wG, wB are Weight Parameters their values are $\mathrm{wR}=0.2125, \mathrm{wG}=0.7154, \mathrm{wB}=0.072$ 
2) Histogram equalization: It is equalize the Histogram to enhance the contrast of the image. It has following steps.
a) Form the cumulative Histogram.(Cum)
b) Normalize the value by dividing it by Total number of pixels. (Cum/Total)
c) Multiply these values by the maximum gray level(L) value and round off the value. $($ cum/total $) *(\mathrm{~L}-1)$
d) Map the original value to the result of step a one-to-one correspondence.

3) Median Filter: It is used to remove the Salt and pepper Noise. It has following steps.
a) Read the pixel values.
b) Sort the pixel values.
c) Find Median value.
d) Substitute median value to central values for each mask.

The above mentioned processes are help to remove the noise from SAR images efficiently and produce the noise filtered SAR image as a output.

\section{B. Edge Detection}

Canny Edge detection [4] is used in this process. Initially noise filtered images was smoothed followed by compute the edge map gradient. To find the Sharp edges, Non-maximum suppression is used. Double thresholding helps to extract the strong and weak edges. To remove those weak edges hysteresis is applied and finally produces the edge image. These processes are illustrated in the following figure 3 .

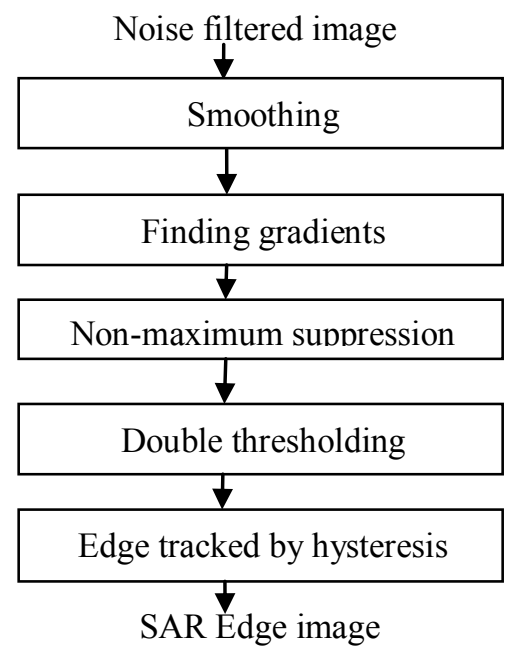

Fig. 3. Edge Detection 
1) Smoothing: The image is smoothed by following equation.

$g(m, n)=G_{\sigma}(m, n) * f(m, n)$

Here $G_{\sigma}=\frac{1}{\sqrt{2 \pi \sigma^{2}}} \exp \left[-\frac{m^{2}+n^{2}}{2 \sigma^{2}}\right]$, where $\sigma=$ Standard deviation. Here $\sigma=1.4, \mathrm{f}(\mathrm{m}, \mathrm{n})$ is noise filtered image.

2) Finding gradients: Gradient magnitude can be determined by the following formula.

$$
M(n, n)=\sqrt{g_{m}^{2}(m, n)+g_{n}^{2}(m, n)}
$$

Here $g_{m}, g_{n}$ are gradient in $x$ and $y$ direction.To finds the direction of the edges by using the following Formula.

$$
\theta(m, n)=\tan ^{-1}\left[g_{n}(m, n) / g_{m}(m, n)\right]
$$

3) Non-maximum suppression: It is used to convert the gradient magnitudes to "sharp" edges. It has the following process.

a) Compare the edge strength of the current pixel with the edge strength of the pixel in the positive and negative gradient direction

b) If the edge strength of the current pixel is largest; preserve the value of the edge strength. If not remove the value

4) Double thresholding: It is used for finding Strong and Weak edges. It has the following steps.

a) Edge pixels stronger than the high threshold are marked as strong. $\mathrm{M}(\mathrm{x}, \mathrm{y})=\mathrm{M}(\mathrm{x}, \mathrm{y})>\mathrm{T} 1 \rightarrow$ Strong edge.

Here $\mathrm{T} 1$ is high threshold value. Where $\mathrm{T} 1=0.8$.

b) Edge pixels weaker than the low threshold are suppressed. $\mathrm{M}(\mathrm{x}, \mathrm{y})=\mathrm{M}(\mathrm{x}, \mathrm{y})>\mathrm{T} 2 \rightarrow$ removed edge.

Here $\mathrm{T} 2$ is low threshold value. WhereT2 $=0.2$.

c) Edge pixels between the two thresholds are marked as weak. $\mathrm{M}(\mathrm{x}, \mathrm{y})=\mathrm{T} 1<\mathrm{M}(\mathrm{x}, \mathrm{y})>\mathrm{T} 2 \rightarrow$ Weak edge.

5) Edge tracked by hysteresis: It is used to eliminate weak edges and ensure the strong edges. It has the following steps.

a) The edge pixels are divided into 8-connected Neighbourhood.

b) It's containing at least one strong edge pixel are then preserved, while other are suppressed.

The above processes are helps to produce an edge image as an output. These edge images also contain some unwanted features. So to extract those linear features (i.e. roads, ridges) CGVF Snake model is proposed in this paper.

\section{Proposed methodology for linear feature extraction}

To extract the linear feature is difficult process in many existing algorithm such as Snakes [1] and GVF Snake [2] algorithm. In particular, they may be not pulled to the 
target feature correctly. So the CGVF Snake model is proposed to improve existing algorithms for extracting linear features and remove the discontinuities efficiently.

Initially GVF field is produced (i.e. internal and external forces of an image) followed by the Snake is created and then CGVF Snake [5] is employed to close the gap (i.e. remove the discontinuities) and finally produces the linear features extracted image. These processes are illustrated in Figure 4.

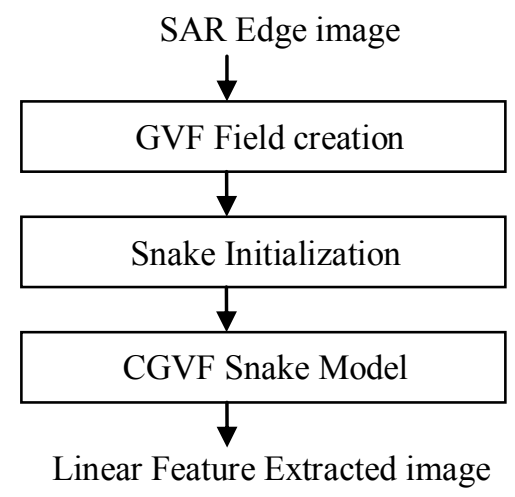

Fig. 4. Linear Feature extraction

1) GVF Field creation: It is defined as the vector field $\mathrm{V}=(\mathrm{u}, \mathrm{v})$. This vector field is created by the following equation.

$$
\begin{aligned}
& \mu \Delta^{2} u-\left(u-f_{x}\right)\left(f_{x}^{2}+f_{y}^{2}\right)=0 \\
& \mu \Delta^{2} v-\left(v-f_{x}\right)\left(f_{x}^{2}+f_{y}^{2}\right)=0
\end{aligned}
$$

Where $f_{x}$ and $f_{y}$ are partial derivative of edge image. $\Delta^{2}$ is Laplacian operator. $\boldsymbol{\mu}$ is used to find the smooth of force field .

2) Snake Initialization: snakes are computer-generated curves that move within images to find object boundaries.

$$
c(s)=(x(s), y(s)), s[0,1] .
$$

Its energy functional can be written as

$$
E_{\text {snake }}=\int_{0}^{1}\left[E_{\text {int }}(c(s))+E_{\text {image }}(c(s))+E_{\text {con }}(c(s))\right] d s
$$

Here $E_{\text {int }}$ - internal energy of the snake, $E_{\text {image }}$ - image forces, $E_{\text {con }}$-external constraint forces.

After initial Snake created then it is moved in image through internal energy, external energy and image force.

3) CGVF Snake Model (proposed method): It is an improved GVF snake model. It is developed from GVF Snake [2]. In GVF snake, snakes are moved in undesired path in edges. It's also generating ears. So the CGVF Snakes are employed to remove the ears and passed through edges in correct direction. It is developed by the following external constrains forces created in this paper. 
a) Point pin energy: It is fixes specified points $\mathrm{P}$ on the Snake. Collection of these connected points to form a basic shape of the Snake. it's energy function can be written as

$$
E_{p i n}=\kappa \sum_{\boldsymbol{p} \in \boldsymbol{P}} \prod_{c_{i} \in \boldsymbol{c}} H\left(\left\|\boldsymbol{c}_{i}-\boldsymbol{p}\right\|-\delta\right)
$$

Here $\kappa$-weight parameter, $\delta$-is the tolerance whether two points are the same or not and $c_{i}$ - Snake points and $H$-Heaviside function is determined by

$$
H(z)=\left\{\begin{array}{l}
1, \text { if } z \geq 0 \\
0, \text { else }
\end{array}\right.
$$

b) Ear avoiding energy: it makes the curve smoother and grows in the correct direction. it's energy function can be written as

$$
E_{\text {ear }}=\rho\left[1-\prod_{\boldsymbol{c}_{i}, c_{i} \in c_{,} \neq j} H\left(\left\|c_{i}-c_{j}\right\|-\delta\right)\right]
$$

Here $\rho$ - Weighted Parameter. $c_{\mathrm{i}}$ and $\mathrm{c}_{\mathrm{j}}$ are start and end points of snake.

External forces are formed by $E_{c o n}=E_{\text {pin }}+E_{\text {ear }}$

This $E_{c o n}$ pixels values are subtituted in equation (7) for snake deformation.

These above mentioned processes are used for extract the linear features. For each itration the snake is grow in edges correct direction and close the gap(i.e. remove the discontinuities) to extract the linear features efficiently. Further improving the feature extraction from SAR image Beamlet Transform also presented in this paper.

\section{Image Enhancement}

To improve the background lighting variation of the SAR image, Image Enhancement [6] process was applied. It helps to eliminate the Non-Uniform background of image and separate features from background thresholding were applied. These processes are illustrated in Figure 5.

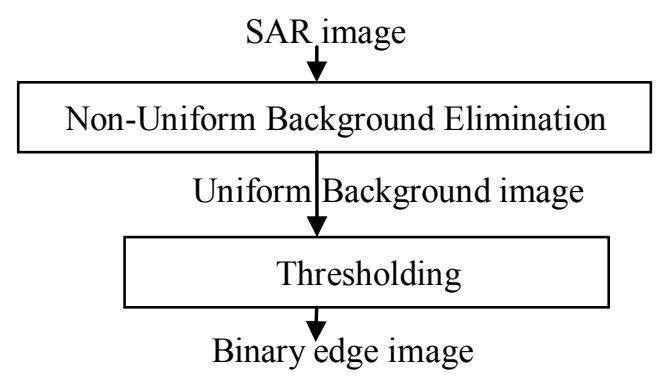

Fig. 5. Image Enhancement

1) Non-Uniform Background Elimination: the uniform background of image was produced by calculating the multiplication factors that eliminate the background lighting variations. It has the following steps 
a) Divide the image into rectangular windows. The image base is $256 * 256$, and the window size is set to $16 * 16$ pixels.

b) Calculate the mean, minimum, and maximum gray level of each window. Denote them as $G_{\text {mean }}, G_{\min }$ and $G_{\max }$.

c) Set an upper limit and a lower limit, for which the points with gray levels outside the limitations are considered as suspicious points for noise, crack, or other objects on the road.

$$
\begin{gathered}
r_{l}=G_{\text {mean }}-\left(G_{\text {mean }}-G_{\text {min }}\right) * 30 \% \\
r_{h}=G_{\text {mean }}+\left(G_{\text {max }}-G_{\text {mean }}\right) * 30 \%
\end{gathered}
$$

d) With the exemption of the suspicious points, recalculate the mean value of the gray level $\mathrm{G}_{\text {mean }}$ '.

e) The amplitude factor correction is calculated as $\mathrm{f}=\mathrm{B} / \mathrm{G}_{\text {mean }}$, thus the modified picture intensity value is defined as $I^{\prime}=I^{*} f$.

2) Thresholding: It is used to separate features(i.e. road or crack edge pixels )from uniform background image. It is defined as

$$
T=\text { Mean- }-(\text { Mean-min }) * 50 \% \text {. }
$$

If gray value $\mathrm{I}(\mathrm{x}, \mathrm{y})<\mathrm{T}$ then set value $\mathrm{I}(\mathrm{x}, \mathrm{y})=0$ else set value $\mathrm{I}(\mathrm{x}, \mathrm{y})=1$;

These above process are helps to enhance the image and produce the binary edge image.

\section{E. Beamlet Transform (proposed method)}

The binary edge image contains linear or curvilinear features. These features are extracted by Beamlet Transform based algorithms were proposed in this paper. The JBEAM tool [8] was performs the Beamlet Transform operation.

Initially the enhanced image was partitioned followed by beamlet dictionary (i.e. tree based Beamlet Representation) is formed by applying the beamlet coder [8] and each beamlets are normalized. thresholding and testing was remove the undefined beamlets and produces the beamlet edge image. These processes are illustrated in Figure 6.

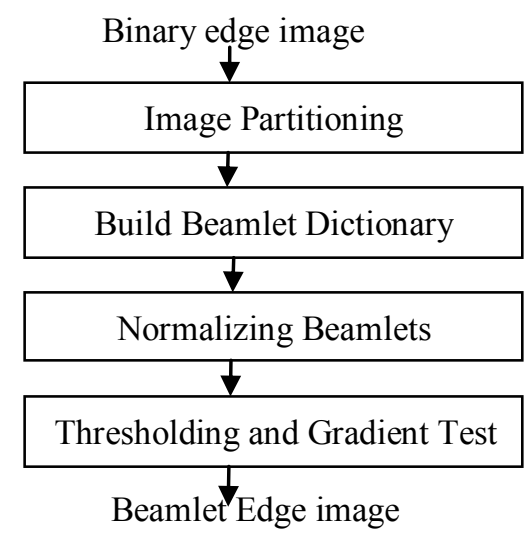

Fig. 6. Beamlet Transform 
1) Image Partitioning: The first stage is partitioning the image into dyadic squares, marking the boundaries of squares with equi-spaced vertices at pixel spacing.

2) Build Beamlet Dictionary: The beamlet dictionary is built by connecting pairs of vertices within a dyadic square. Beamlet coder was build the tree based beamlet representation (BR).here the length and orientation of beamlet was calculated by converting BR into Symbol Stream and reconstructs the symbol stream into Beamlets.

3) Normalizing Beamlets :the beamlets are normalized by the following equation

$$
T_{f}^{\prime}(b)=T_{f}(b) / \sqrt{L(b)}
$$

Here $\mathrm{T}_{\mathrm{f}}(\mathrm{b})$-beamlet co-efficient ,L(b)-length of the beamlet.

4) Thresholding and Gradient Test: The normalized beamlets are compared with threshold value is given by

$$
T=\left[\max \left(T_{f}^{\prime}(b)\right)-\min \left(T_{f}^{\prime}(b)\right)\right] / 2
$$

If $\mathrm{T}_{\mathrm{f}}{ }_{\mathrm{f}}(\mathrm{b})$ is greater than $\mathrm{T}$, a line segment is considered to exist in the sub-square, if $\mathrm{T}_{\mathrm{f}} \mathrm{f}(\mathrm{b})$ is less than the threshold value, the beamlet is discarded. Finally test whether all sub-square are connected or not.

These above process are helps to produces the beamlet edge image. These images also contain some discontinuities.

\section{F. Linear Feature Extraction}

After the Beamlet Transform, beamlet edge image contains some discontinuities To extract the linear features and remove discontinuities post processing algorithm was developed in this paper. The post processing includes edge linking and thinning process [9] was illustrated in Figure 7.

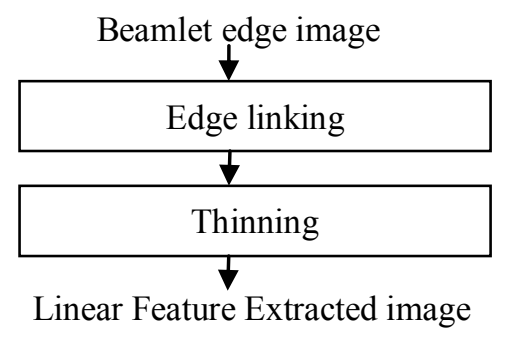

Fig. 7. Linear Feature Extraction

1) Edge Linking: It is used to link the discontinuity edges. It has the following steps.

a) Find the gradient in $x$ and $y$ direction (i.e $G_{x}$ and $G_{y}$ ).

b) Edge linking is performed by the following equation

$$
I(x, y)=\operatorname{sqrt}\left(G_{x}^{2}+G_{y}^{2}\right)
$$

Here $\mathrm{I}(\mathrm{x}, \mathrm{y})$-edge linked beamlet image. 
2) Thinning: It is used to remove the unwanted beamlets and produces the linear feature extracted image.

These above process are helps to extract the linear features efficiently and performances of the images are evaluated in this paper.

\section{G. Performance Evaluation}

The performance [10] of the image is calculated by the following process.

1) Peak Signal to Noise Ratio(PSNR):The PSNR is used to find the difference between two images. It is defined as

$$
P S N R=20 * \log 10(\mathrm{~b} / \mathrm{MSD})
$$

Where $b$ is the largest value of the signal (typically 255or 1)

and MSD is mean square difference between two images.

$$
M S D=\frac{\sum_{r, c}\left(I_{s}(r, c)-I_{d}(r, c)\right)^{2}}{R \times C}
$$

Where $\mathrm{I}_{\mathrm{s}}(\mathrm{r}, \mathrm{c})$ - noisy image. $\mathrm{I}_{\mathrm{d}}(\mathrm{r}, \mathrm{c})$-denoised image. $\mathrm{R}, \mathrm{C}$ - number of rows and columns respectively.

2) Noise Mean Value (NMV): It determines the quantity of noise content in the denoised image. Lower value means lower noise content. It is defined as

$$
N M V=\frac{\sum_{r, c} I_{d}(r, c)}{R \times C}
$$

3) Noise variance $(N V)$ : It determines the content of noise in the image. The lower variance gives cleaner image as more noise is reduced.

$$
N V=\frac{\sum_{r, c}\left(I_{d}(r, c)-N M V\right)^{2}}{R \times C}
$$

\section{Experimental Results}

The proposed CGVF Snake model has been implemented and the performance results are presented. Figure(8) shows an TRA_SAR_image1 represents the road netwok and Figure (9) shows an TRA_SAR_image2 represents the montain area.These images are taken from china with high resolution and high frequency.

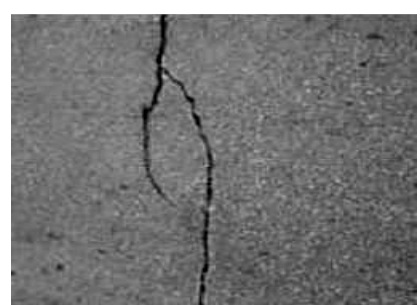

Fig. 8.

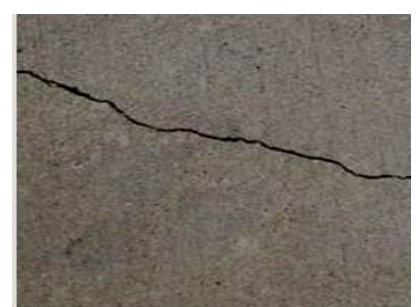

Fig. 9. 
During the Noise Removal process, Noise removed image is produced. Figure(10) and Figure(11) shows an noise removed image1\&2 for TRA_SAR_IMAGE1 and TRA_SAR_IMAGE2.Here size of images are $256 \times 267$ pixel and $[3 \times 3]$ median filter is used in this process.

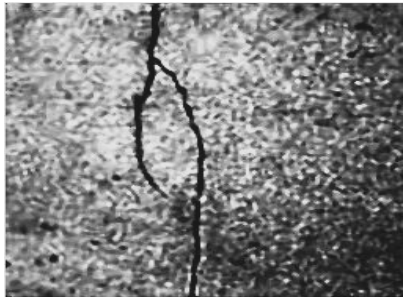

Fig. 10 .

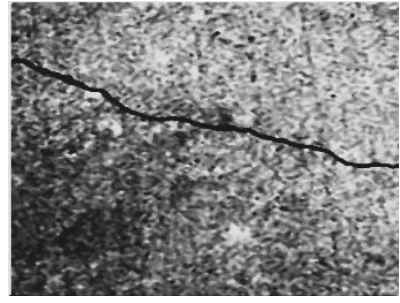

Fig. 11.

During the edge detection process, edge image is produced. Figure(12) and (13) shows an Edge image1\&2.

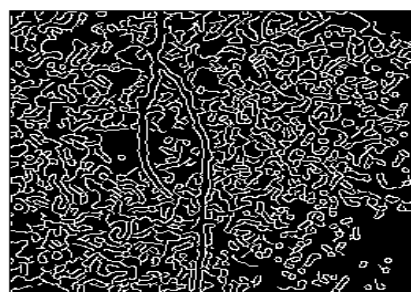

Fig. 12.

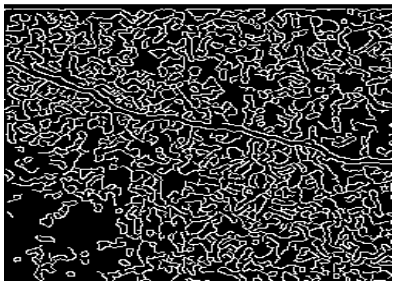

Fig. 13.

During linear feature extractoion process, linear feature extracted image is produced.Figure(14) and (15) shows an Linear Feature Extracted image1\&2.for GVF creation process , $\mu$ is set to be 0.2 and iteration $=80$.

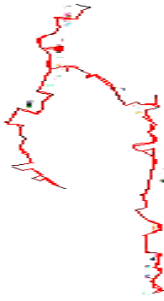

Fig. 14.

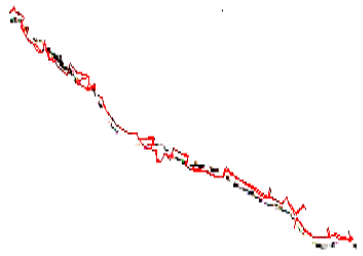

Fig. 15.

Linear Feature Extraction based on Beamlet Transform has been implemented and performance results are presented. During Edge Enhancement process, binary edge image is produced. Figure (16) and Figure (17) shows binary edge image1\&2.here threshold $\mathrm{T}=0.43$ for binary edge image 1 and $\mathrm{T}=0.45$ for binary edge image 2 . 


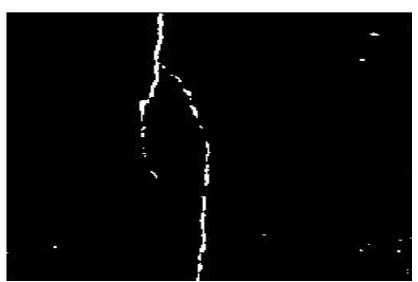

Fig. 16.

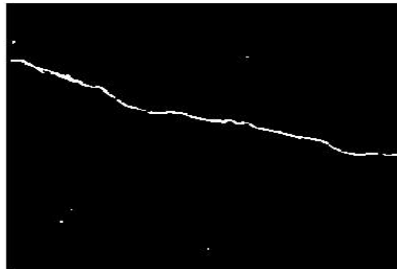

Fig. 17.

During Beamlet Transform process, Beamlet edge image is produced. Figure (18) and Figure (19) shows Beamlet edge image1\&2.size of images should be $256 \times 256$ pixels.

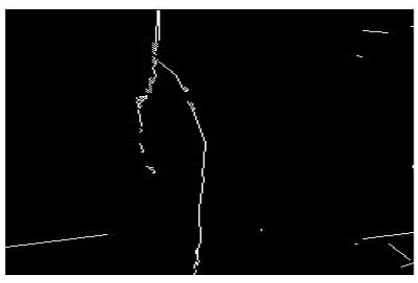

Fig. 18.

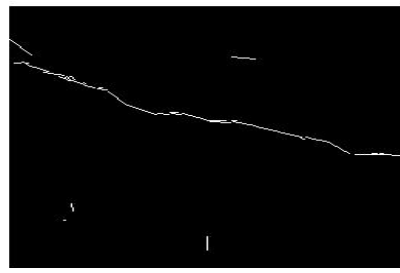

Fig. 19.

During Linear Feature Extraction process, linear feature extracted image is produced. Figure (20) and Figure (21) shows linear Feature extracted image1\&2. Size of images should be $256 \times 256$ pixels.

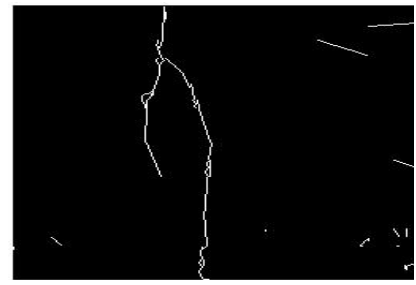

Fig. 20.

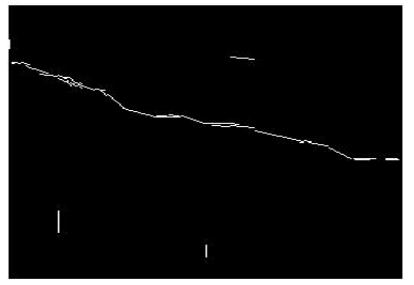

Fig. 21.

The Performance of the CGVF Snake Model and beamlet transform were evaluated and corresponding results are Shown in Table I and Table II.

Table 1. Performance for CGVF Snake Model

\begin{tabular}{|c|c|c|c|c|c|c|}
\hline $\begin{array}{c}\text { Figure } \\
\text { Name }\end{array}$ & NMV & NV & MSD & M & $\begin{array}{c}\text { Itera } \\
\text { Tion }\end{array}$ & $\begin{array}{c}\text { PSNR } \\
(\text { db })\end{array}$ \\
\hline $\begin{array}{l}\text { SAR_ } \\
\text { IMAGE1 }\end{array}$ & 125.55 & 4851.68 & 5729.7 & 0.2 & 100 & 10.583 \\
\hline $\begin{array}{c}\text { SAR_ } \\
\text { IMAGE2 }\end{array}$ & 127.06 & 4669.35 & 3360.3 & 0.2 & 100 & 12.90 \\
\hline
\end{tabular}


Table 2. Performance for Beamlet Transform

\begin{tabular}{|c|c|c|c|c|c|c|}
\hline $\begin{array}{c}\text { Figure } \\
\text { Name }\end{array}$ & NMV & NV & MSD & T & $\begin{array}{c}\text { No of } \\
\text { Nonzeros }\end{array}$ & $\begin{array}{c}\text { PSNR } \\
(\mathrm{db})\end{array}$ \\
\hline $\begin{array}{c}\text { SAR_- } \\
\text { IMAGE1 }\end{array}$ & $\mathbf{8 3 . 9 1 0}$ & 76.456 & 123.877 & 0.42 & 944 & 27.234 \\
\hline $\begin{array}{c}\text { SAR_- } \\
\text { IMAGE2 }\end{array}$ & 112.599 & 112.599 & 363.552 & 0.45 & 707 & 22.559 \\
\hline
\end{tabular}

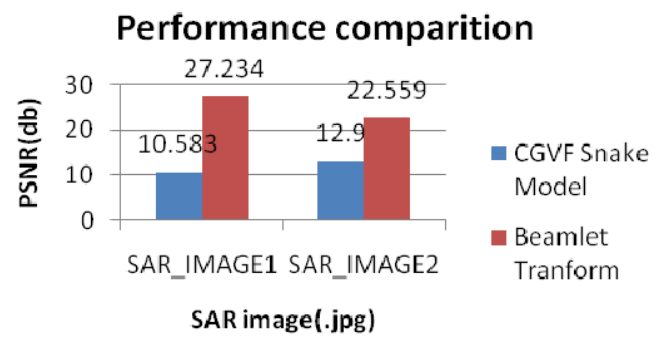

Fig. 22. BAR Graph(performance comparition)

Figure. 22 shows the BAR Graph.Here the PSNR value for Beamlet Transform was high compare to the CGVF Snake Model.Hence, from the BAR Graph, the performance of the beamlet Transform based algorithm gives best results compared to the CGVF Snake model.

\section{Conclusions}

Linear Feature is an important characteristic in Microwave images. These images are affected by noise. So the noise is removed in this work by using median filter method. After that the edges are detected efficiently by applied a canny edge detection algorithm.GVF field is produced and then Snake was deformed in edges by applying an external constrain forces of CGVF Snake model. After Snake deformation was completed, the linear features are extracted. Non-uniform background elimination process helps to enhance the image followed by applied the beamlet transform to extract linear features and discontinuities are removed in this paper. Finally CGVF Snake Model and Beamlet Transform are compared and experimental results are presented. From the experimental results, beamlet transform is the best approach for extracting the linear feature efficiently and increase quality of satellite Mapping. In future work, the new algorithm has to be implemented for improving the extraction of linear feature from microwave images to overcome the problems of missing edges in this work. 


\section{References}

[1] Kass, M., Witkin, A., Terzopoulos, D.: Snakes: active contour models. International Journal of Computer Vision 1, 321-331 (1987)

[2] Xu, C., Price, J.L.: Snakes, shapes, and gradient vector flow. IEEE Transactions on Image Processing 7, 359-369 (1998)

[3] Min, W., Yanning, Z.: Gray-scale Image Edge Detection Based on directional Beamlet Transform. IEEE Transactions on Image Processing 7, 1120-1124 (2008)

[4] Canny Edge Detection (May 23, 2009), http://www.cse.iitd.ernet.in/ pkalra/csl783/canny.pdf

[5] Deng, X.-P., He, C., Sun, H.: An Improved GVF Snake Model and its Application to Linear Feature Extraction from SAR Images. IEEE. ICSP, 2063-2067 (2010)

[6] Ying, L., Salari, E.: Beamlet Transform Based Technique for Pavement Image Processing and Classification. IEEE Transactions on Image Processing, 141-145 (2009)

[7] Salari, E., Zhu, Y.: A Road Extraction Method Using Beamlet Transform. IEEE Transactions on Image Processing, 1130-1134 (2012)

[8] Huo, X., Chen, J.: JBEAM: multi scale curve coding via beamlets. IEEE Trans. Image Processing 14, 1665-1677 (2005)

[9] Edge Thinning Used in the SUSAN Edge Detector, http://users.fmrib.ox.ac.uk/ steve/susan/thinning/ thinning.html

[10] Joshi, R., Garg, R.D.: Pre-processing of TerraSAR-X Data for Speckle Removal: An Approach for Performance Evaluation. Indian Soc. Remote Sens. 40(3), 371-377 (2012)

[11] Wei, N., Zhao, X., Dou, X., Song, H.: Beamlet Transform Based Pavement Image Crack detection. In: IEEE International Conference on Intelligent Computation Technology and Automation, vol. 13, pp. 881-883 (2010) 